\title{
Modelle zur Definition von Transsexualität und ihre Auswirkungen auf die gesellschaftliche Akzeptanz - Das Beispiel Stimme und Sprechverhalten
}

Christiane Neuschaefer-Rube, David Scheidt, Dominik Groß

\section{Einleitung}

In den letzten Jahrzehnten hat das interdisziplinäre Forschungsgebiet „Gender Studies“ maßgeblich zu einer Erweiterung und Flexibilisierung der Diskurse über die Kategorie Geschlecht beigetragen. Dementsprechend hat sich auch der Blick auf Menschen, die sich nicht dem Geschlecht zugehörig fühlen, das ihnen bei ihrer Geburt aufgrund genetischer und phänotypischer Merkmale zugeschrieben wurde („Transsexuelle“, „transidente Personen“ bzw. „Transgenders“), ${ }^{1}$ unter dem Einfluss der Gender-Forschung maßgeblich verändert. Andererseits wird der gesellschaftliche Umgang mit Transsexualität immer noch stark geprägt durch die „Deutungsmacht“ Medizin, die das Phänomen als Störung der Geschlechtsidentität einordnet und ihr einen Krankheitscharakter zuschreibt.

Der vorliegende Aufsatz versucht nach einem konzisen Überblick über die verfügbaren Modelle zur Definition von Geschlecht (Kapitel 2) den möglichen Widerspruch zwischen dem medizinischen Konzept von Transsexualität (Kapitel 3) und der Selbstwahrnehmung vieler Betroffener offen zu legen (Kapitel 4). Sodann gilt es mit Hirschauers Modell einer interaktiven Konstruktion

1 Zur Terminologie vgl. den Beitrag von Steinmetzer/Groß in diesem Band, hier insb. Fußnote 2 sowie Kapitel 4 dieses Beitrags. Vgl. weiterhin Scheidt (2003); Steinmetzer/Groß (2006); Steinmetzer/Groß/Duncker (2007); Groß/ Steinmetzer (2007). 
von Geschlechtszugehörigkeit (Kapitel 5) einen Weg zu einem alternativen Umgang und damit zur „Depathologisierung“ von Transgenders aufzuzeigen. Hirschauer begreift Geschlecht letztlich als eine Kategorie, die aktiv gestaltet bzw. beeinflusst werden kann. Dabei wird der (durchaus variablen) Attribuierung von Geschlechtszugehörigkeit besondere Bedeutung beigemessen. Im Anschluss daran wird am Beispiel von Frau-zu-Mann-Transsexuellen das Potential ausgelotet, das Hirschauers Konzept im Hinblick auf eine aktive Veränderung der Geschlechtsdarstellung eröffnet (Kapitel 6). Schließlich wird - vor dem Hintergrund der Tatsache, dass der Stimme in der Regel eine wichtige Rolle in der Geschlechtsdarstellung eines Menschen zugeschrieben wird - dieses Modell auf den Bereich der Stimmarbeit angewandt und sein Potential für die Anpassung von Stimme und Sprechverhalten aufgezeigt (Kapitel 7).

\section{Modelle zur Definition von Geschlecht: Ein kurzer Überblick}

Geschlecht lässt sich als eine natürliche Gegebenheit oder als ein komplexes mehrdimensionales Gebilde betrachten. Als Hilfestellung bietet die englische Sprache zwei Termini für Geschlecht an: „sex“ und „gender“. Dabei wird sex im Sinne von „körperliches“ oder „biologisches Geschlecht"“2 verstanden:

„the biological qualities that distinguish between male and female. These qualities are expressed by an individual's chromosomal, gonadal, morphological (internal and external) and hormonal characteristics." ${ }^{33}$

Demgegenüber entspricht gender am ehesten der deutschen Umschreibung "soziales oder gelebtes Geschlecht" und meint den Aspekt von Geschlecht, der durch eine Zuschreibung von Seiten der Gesellschaft (Geschlechterrolle) und das eigene Zugehörigkeitsgefühl (Geschlechtsidentität) realisiert wird. ${ }^{5}$ Innerhalb der Geschlechterforschung hat man diese Begriffsdualität aufgegriffen und dahingehend erweitert, dass nicht nur gender, sondern auch „der vermeintlich natürliche Körper (sex)“ als „soziokulturelle Konstruktion“ zu betrachten ist: ${ }^{6}$

„Aus biologischen Parametern entstehen aus dieser Sicht noch keine Geschlechter und keine Geschlechterordnung - aber aus einer Geschlechterordnung können biologische Merkmale zu Geschlechtszeichen und zu einem zentralen Bedeutungsgehalt werden. ${ }^{~}{ }^{7}$

Die Geschlechterdifferenz ist somit „nicht mehr an den Geschlechtskörper gebunden. “8 Hinsichtlich der Zuordnung einer konkreten Person zu einem bestimmten Geschlecht ist in den meisten menschlichen Gesellschaften die Annahme einer von der Natur vorgegebenen Zweiteilung von Geschlecht vor-

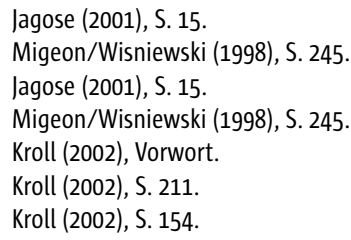


herrschend: ${ }^{9}$ Für jeden Menschen gibt es demnach ein eigenes und ein anderes Geschlecht und ein Gefühl der Geschlechtszugehörigkeit (,ich bin ein Mann und keine Frau“), das selbstverständlich zum eigenen Geschlecht passt. ${ }^{10}$ Dabei werden die körperlichen Ebenen von Geschlecht, d.h. die äußeren und inneren Geschlechtsorgane, als entscheidend dafür angesehen, welches der beiden Geschlechter als das eigene oder das andere angesehen wird. Kessler und McKenna (2000) fassen diese Sichtweise von Geschlecht folgendermaßen als „taken-for-granted beliefs of the culture“"11 ${ }^{\text {zusammen: }}$

„1. There are two and only two genders. [...]

2. Gender exists as a biological, fact' independently of anyone's ideas about gender.

3. A person's gender never changes.

4. Genitals are the essential defining feature of gender [...].“

Man kann davon ausgehen, dass die Geschlechtermodelle der meisten Menschen, d.h. auch der Ärzte, Psychologen und Logopäden, grundsätzlich mit diesen Überzeugungen übereinstimmen. ${ }^{12}$ Das bedeutet, dass auch die Definition, Diagnostik und Therapie von Geschlechtsidentitätsstörungen auf der Basis des Primats der Zweigeschlechtlichkeit, der Unwandelbarkeit von Geschlecht, der Dominanz des körperlichen über das soziale Geschlecht sowie der Selbstverständlichkeit des Übereinstimmens der verschiedenen Dimensionen von Geschlecht entwickelt wurden.

\section{Medizin als Deutungsmacht? Zur Frage der Pathologisierung von „Transsexualität“}

Transsexualität oder Transsexualismus ist eine vorrangig im medizinischen Diskurs verwendete Bezeichnung bzw. Diagnose für Menschen, die den Wunsch haben, „als Angehörige[r] des anderen anatomischen Geschlechts zu leben und anerkannt zu werden. Dieser geht meist mit dem Gefühl des Unbehagens oder der Abgrenzung hinsichtlich des eigenen Geschlechts einher. " ${ }^{\text {13 }}$ Die International Classification of Diseases, Version 10 (ICD-10) ordnet den Transsexualismus (F 64.o) im Kapitel V (F) „Internationale Klassifikation

9 Hirschauer (1999, S. 21) verweist auf „kulturanthropologische Untersuchungen, die eine kulturelle Relativität von Geschlechtsklassifikationen aufzeigten".

10 Sigusch (1997) bezeichnet diesen Zustand als „Zissexualismus“ und „geschlechtseuphorisch“, wenn „Körpergeschlecht, Geschlechtsrolle und Geschlechtsidentität fraglos und scheinbar natural zusammenfallen“ (S. 871) [im Gegensatz zu „Transsexualismus“ bzw. „Geschlechtsdysphorie“].

11 Kessler/McKenna (2000), S. 2.

12 Ausnahmen stellen körperliche Erscheinungsweisen dar, die im medizinischen Kontext als „Intersexualität“ bezeichnet werden. Gemeint ist damit der „Zustand eines Individuums mit Widersprüchen in der Ausbildung der allgemeinen äußeren geschlechtlichen Erscheinung [...], der Keimdrüsen bzw. Geschlechtsorgane [...] sowie des chromosomalen Geschlechts“; Roche Lexikon Medizin (1987), S. 882.

13 Clement/Senf (1996), S. 2. Dabei werden als „Mann-zu-Frau-Transsexuelle“ diejenigen Menschen bezeichnet, deren Geburtsgeschlecht als männlich bezeichnet wurde und die ihr empfundenes Geschlecht als weiblich angeben, wohingegen „Frau-zu-Mann-Transsexuelle“ bei der Geburt als Mädchen eingestuft wurden und sich dem männlichen Geschlecht zugehörig fühlen. 
psychischer Störungen“ als „Störung der Geschlechtsidentität“ (F 64) unter die Persönlichkeits- und Verhaltensstörungen (F 6) ein. ${ }^{14}$

Die Kategorisierung der Transsexualität als psychisches Leiden mit Krankheitswert hat für die Betroffenen den entscheidenden Vorteil, dass die medizinischen Maßnahmen zur Linderung des Leidensdrucks (Psychotherapie, Hormone und die wichtigsten Operationen) bei entsprechendem Nachweis in Deutschland von den Krankenkassen bezahlt werden müssen. Nachteile bestehen in der Abhängigkeit von ärztlicher Begutachtung und Verordnung und der damit verbundenen Pathologisierung des transsexuellen Empfindens. Die Klassifikation als psychiatrisches Störungsbild kann sowohl Auswirkungen auf das Selbstbild der Betroffenen haben (,,ich leide an einer psychischen Störung“) als auch Berührungsängste im gesellschaftlichen Umfeld erzeugen.

Dabei stellt sich die Frage nach der Begründung für die mit der Einordnung als psychische Störung einhergehende Pathologisierung der Transsexualität. In Bezug auf die oben erwähnten Annahmen über Geschlecht stellt das Phänomen Transsexualität in vielerlei Hinsicht eine Abweichung von der Norm dar: Das Zugehörigkeitsempfinden zu einem Geschlecht und die biologische Geschlechtsdarstellung fallen auseinander, ersteres wiegt für die Betroffenen schwerer als das, was ihr Körper darstellt, und mit dem Wunsch nach hormoneller und chirurgischer Geschlechtsumwandlung rütteln sie am Prinzip der Unwandelbarkeit von Geschlecht.

Ab wann die Normabweichung als pathologisch und damit behandlungsbedürftig anzusehen ist, ist schwer zu beantworten. Im Fall der abweichenden Geschlechtsidentität scheinen jedoch tragende Pfeiler der Gesellschaft (wie z. B. die Rollenaufteilung zwischen den Geschlechtern in Bezug auf Arbeit und Fortpflanzung) zur Disposition zu stehen, so dass eine Tendenz besteht, gesamtgesellschaftliche Erschütterungen zu verhindern, indem ein Krankheitsbild geschaffen wird, das zumindest das Prinzip der Zweigeschlechtlichkeit bestehen lässt. Stefan Hirschauer (1999) spricht in diesem Zusammenhang von der „sozialen Konstruktion der Transsexualität“ und vertritt die These, „daß die medizinische Konstruktion der Transsexualität ein immanenter Bestandteil der zeitgenössischen Konstruktion der Zweigeschlechtlichkeit ist. In dieser These sind zwei weitere enthalten: daß die Transsexualität vor allem durch die Medizin selbst hervorgebracht wird; und daß die Geschlechtszugehörigkeit von Gesellschaftsmitgliedern eine durch und durch soziale Konstruktion

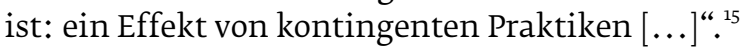

Da ein transsexueller Mensch im Unterschied zum intersexuellen per definitionem eine eingeschlechtliche körperliche Differenzierung aufweist, sind für die Erstellung von Diagnose, Differentialdiagnose und Therapieindikation psychiatrisch-psychotherapeutisch tätige Ärzte zuständig. Diese Zuständigkeit beinhaltet jedoch keine grundsätzliche Gleichsetzung der Diagnose Transsexualität mit psychiatrischer Komorbidität oder Behandlungsbedürftigkeit. Vielmehr scheint es aufgrund der Vielfalt der individuellen Erscheinungsbilder sehr schwierig zu sein, von psychiatrischer Seite dem Phänomen Trans-

14 Huber (1999), S. 720.

15 Hirschauer (1999), S. 9. 
sexualität und der Situation der Betroffenen gerecht zu werden. So bezeichnet Sigusch (1997) das Auseinanderklaffen von Geschlechtsidentität und körperlichem Geschlecht nach wie vor als „Rätsel“: Es sei „nicht gelungen, die Psychound Soziogenese transsexueller Entwicklungen ätiopathogenetisch auf einen ebenso spezifischen wie überzeugenden Nenner zu bringen“. ${ }^{16}$

Eine kausale Therapie der Transsexualität im Sinne einer Korrektur des gegengeschlechtlichen Zugehörigkeitsgefühls ist nach verbreiteter Auffassung nicht möglich; eine solche Möglichkeit kann aber auch nicht ausgeschlossen werden. Dennoch sind Ärzte und andere mit der Transsexualität befasste Berufsgruppen (z. B. Psychologen, Logopäden, Amtsrichter) aufgefordert, den Betroffenen Hilfestellungen anzubieten.

Seit einiger Zeit erfolgt das medizinische und rechtliche Vorgehen in Deutschland standardisiert: über die sogenannten „Standards der Behandlung und Begutachtung von Transsexuellen“ (deutsche „Standards of Care“) (17 $^{17}$ bzw. das „Gesetz über die Änderung der Vornamen und die Feststellung der Geschlechtszugehörigkeit in besonderen Fällen“ („Transsexuellengesetz“ [TSG]). ${ }^{18}$ Dabei gehören zu den medizinischen Maßnahmen der Geschlechtsangleichung die psychotherapeutische Begleitung, die gegengeschlechtliche Hormonbehandlung sowie verschiedene Transformationsoperationen.

\section{Der Gegenentwurf: Selbstwahrnehmung und Selbstbeschreibung der Betroffenen}

Menschen, die sich mit dem Geschlechtseintrag, der zum Zeitpunkt ihrer Geburt vorgenommen wurde, und den damit verbundenen Attribuierungen bzw. Rollenerwartungen nicht identifizieren können, definieren sich häufig selbst nicht so, wie es die ICD-1o beschreibt. Zum einen schreiben sie der eigenen „Transsexualität“"keinen Krankheitscharakter zu, d. h. sie akzeptieren die oben skizzierte Pathologisierung durch die Medizin nicht; zum anderen kommt es vor, dass sie sich weder als Frau noch als Mann oder sowohl weiblich als auch männlich fühlen und deswegen unter der in der ICD-10 vorgenommenen eindeutigen Zuschreibung zu einem Geschlecht leiden. Für die Behandlung kann das bedeuten, dass z. B. die Verabreichung von Hormonen gewünscht wird, jedoch keine operative Geschlechtsangleichung oder nur ein Teil der chirurgischen Maßnahmen.

Das Tragische an der Situation der Betroffenen ist die Schwierigkeit oder manchmal auch Unmöglichkeit, in einer zweigeschlechtlich organisierten Gesellschaft mit diesem Lebensentwurf ernst genommen zu werden. Dieses Problem stellt sich sowohl bei den Anlaufstellen für die Einleitung medizinischer oder rechtlicher Maßnahmen als auch unter den Betroffenen selbst und äußert sich z. B. in Zweifeln über das Ausmaß des Leidensdruckes, an der Berechtigung des Zugangs zu medizinischen Maßnahmen und in der Umdeutung derselben von einer Heilmaßnahme zur Schönheitskorrektur.

16 Sigusch (1997), S. 871; Sigusch (2005), S. 139.

17 Becker et al. (1997).

18 Sigusch (1997), S. 875 . 
Infolgedessen sind die meisten Betroffenen, die auf dem offiziellen Weg eine Verbesserung ihrer Lebenssituation in Bezug auf ihre Geschlechtsdarstellung erreichen wollen, versucht, sich den Gutachtern gegenüber zu verstellen, um den Kriterien der Standards of Care zu entsprechen bzw. um nicht abgewiesen zu werden, wenn sie berichten, was sie wirklich empfinden und was sie verändern wollen und was nicht.

Vor dem Hintergrund dieser Tatsache entstand in der medizinischen Literatur ein einheitliches Bild von der Symptomatik der Transsexualität, dem die gegengeschlechtliche Identifizierung und der Wunsch nach vollständiger Geschlechtsangleichung „selbstverständlich“ immanent sind. In anderem Kontext (z. B. in sozialwissenschaftlicher Literatur oder in Alltagsgesprächen mit Betroffenen) hat die Vielfalt der Selbstbeschreibungen im Hinblick auf Geschlecht deutlich mehr Raum. Es gibt Definitionsversuche, die offener gehalten werden, um möglichst wenige Personen auszuschließen:

„Transgender ist ein Begriff mit vielen verschiedenen und oft schwierigen Interpretationsmöglichkeiten. Deshalb wird er in zunehmendem Maße verwandt, weil er all diejenigen mit einbezieht, die den heteronormativen Kategorien von ,Mann' oder ,Frau' nicht entsprechen können oder wollen. [...] Die Begrifflichkeiten sind alle sehr schlecht zu fassen, mein Rat ist, im Zweifelsfall die Person selbst zu fragen, mit welchen Worten sie sich beschreibt.. ${ }^{19}$

Kessler und Mc Kenna beschreiben die sozialen Schwierigkeiten von Menschen, die als Transgender leben:

„Transgendered people - in one way or another - place themselves outside the conventional female/male dichotomy, yet live in a social world that recognizes only females and males. ${ }^{420}$

Transgender haben im Alltag nicht die Möglichkeit, als solche wahrgenommen zu werden, weil es in der Gesellschaft keine Kategorie jenseits von männlich und weiblich gibt.

Vor diesem Hintergrund ist die Bezeichnung „Transgender“ zu verstehen als Sammelbegriff, der auch die Transsexualität als eine idealisierte Art, Geschlechtsidentität zu empfinden und darzustellen, beinhaltet. Darüber hinaus soll diese Bezeichnung auf die Notwendigkeit hinweisen, sich als Behandler, der durch einen Klienten mit dem Thema "Geschlechtsidentität“ in Berührung kommt, mit der jeweils individuellen Selbstbeschreibung und den daraus resultierenden Wünschen nach Veränderung der Geschlechtsdarstellung und -zuschreibung auseinander zu setzen und diese bei Diagnostik und Therapieplanung zu berücksichtigen.

Frau-zu-Mann-Transsexuelle bezeichnen sich häufig als „Transmann“. Die gleichnamige deutschlandweite Interessenvertretung, der Transmann e. V., gibt in einem Flugblatt folgende Beschreibung seiner Zielgruppe: „Für transgender, transidentische, transsexuelle und intersexuelle Männer und alle Menschen, deren Geschlechtseintrag weiblich lautet oder lautete, die

19 Del LaGrace Volcano, in: Schulte-Fischedick (2002), S. 14.

20 Kessler/Mc Kenna (2000), S. 1. 
sich jedoch mit diesem Wort falsch oder unvollständig beschrieben fühlen. “ Dies umfasst gängige Bezeichnungen, mit denen sich Betroffene selbst beschreiben, wobei es unterschiedliche Gründe für die Wahl der einzelnen Formulierungen gibt. Ein erster Anhaltspunkt sind die Schwerpunktsetzungen innerhalb der Begriffe: Als transident(isch) (im Gegensatz zu transsexuell) bezeichnen sich Menschen, die ausdrücken wollen, dass es ihnen in ihrer Selbstbeschreibung vor allem um ihre Geschlechtsidentität und nicht so sehr um ihre Sexualität oder ihr körperliches Geschlecht geht.

\section{Hirschauers Modell einer interaktiven Konstruktion von Geschlechtszugehörigkeit - Ein Weg zur Depathologisierung?}

Im Hinblick auf die beschriebenen Konzeptionen von Geschlecht stellt sich die Frage, wie Transgender ihre empfundene Geschlechtszugehörigkeit, die nicht ihren biologischen Geschlechtsmerkmalen entspricht, zum Ausdruck bringen können. Hierbei scheint Hirschauers Modell einer „interaktive[n] Konstruktion von Geschlechtszugehörigkeit“, die er „als ein Phänomen öffentlicher Geltung in sozialen Beziehungen" begreift, ${ }^{21}$ hilfreich zu sein. Mit diesem Modell widerspricht er dem Alltagsverständnis von einer naturgegebenen „offensichtlichen Geschlechtszugehörigkeit ${ }^{\text {“22 }}$, die weder aufwändig dargestellt noch entziffert werden muss. Wenn Hirschauer demgegenüber von einer konstruierten Geschlechtszugehörigkeit spricht, bedeutet dies, dass er Geschlecht im Sinne eines „Doing gender“'23 versteht, das durch „eine permanente Praxis von Zuschreibungs-, Wahrnehmungs- und Darstellungsroutinen“"24 von Kommunikationspartnern hergestellt wird. Dabei beschreibt Hirschauer die Geschlechtswahrnehmung (nach Kessler/McKenna [1978]) als „einen komplizierten Attributionsprozess“, der „durch das Alltagswissen von der Zweigeschlechtlichkeit gesteuert wird, das Teilnehmer zwingt, entweder Männer oder Frauen zu sehen und außerdem, wenn diese Entscheidung getroffen wurde, die Zuschreibung auch gegen andere ,Evidenzen' fortzusetzen, weil sie eben wissen, dass Personen ihr Geschlecht dauerhaft haben und nicht einfach wechseln können" ${ }^{25}$

Er fügt hinzu, „dass nicht nur Personen, sondern vielen kulturellen Objekten [wie z. B. Kleidungsstücken, Tätigkeiten, Namen, Wörtern etc.] eine Geschlechtsbedeutung zugeschrieben wird“, und nennt diesen Vorgang „Sexuierung “. ${ }^{26} \mathrm{Am}$ überraschendsten ist diese Zuschreibungspraxis in Bezug auf die Genitalien: Sie werden von Geburt an als Zeichen für die Geschlechtszugehörigkeit verwendet, obwohl sie bei Alltagsbegegnungen nicht sichtbar

\footnotetext{
21 Hirschauer (1999), S. 25.

22 Hirschauer (1999), S. 32.

23 Kroll (2002), S. 72.

24 Kroll (2002), S. 211.

25 Hirschauer (1999), S. 27.

26 Ebd.
} 
sind und daher nur als „Insignien“ begriffen werden, „die da sein sollen, und zwar immer schon“. ${ }^{27}$

Die Attribuierung von Geschlechtszugehörigkeit hat für Transgenders viele Vorteile und bietet eine Entlastung bezüglich ihrer Anstrengungen, als das Geschlecht wahrgenommen zu werden, das sie darstellen wollen:

- Wenn Interaktionspartner gezwungen sind, das Geschlecht ihres Gegenübers korrekt und mit Leichtigkeit zu erkennen, werden sie tendenziell über Uneindeutigkeiten in der Darstellung hinwegsehen, um „die Blamage einer Verwechslung ${ }^{\text {(28 }} \mathrm{zu}$ vermeiden.

- Wenn es neben dem Körper auch andere Objekte gibt, denen eine Geschlechtsbedeutung zugeschrieben wird, können Menschen diese auch erfolgreich zur Geschlechtsdarstellung nutzen. Dazu Hirschauer weiter: „es gibt weder eine feste Hierarchie von Geschlechtsmerkmalen noch einen durchgängigen Primat von körperlichen Zeichen. [...] Ferner sind nicht einzelne Indizien, sondern der variable Zusammenhang von Darstellungselementen für Geschlechtsattributionen wichtig“. ${ }^{29}$ Das bedeutet ein gewisses Maß an Gestaltungsfreiheit für die Präsentation von Transgenders und die Möglichkeit des „Ausgleichs“ von „,nachteilige[n]“ Körperformen“ oder „Elemente[n] des ,falschen“ Darstellungsrepertoires“. ${ }^{\circ}$

- Wenn das Vorhandensein von zur Zuschreibung passenden Genitalien nur unterstellt wird, müssen sie weder tatsächlich vorhanden sein noch eine bestimmte Gestalt haben.

Durch die „Erweiterung des Darstellungsbegriffs auf die Geschlechtszugehörigkeit ${ }^{\text {"31 }}$ wird Transgenders die Möglichkeit gegeben, ihre passive Haltung zum Geschlecht als „körperliche[r] Fatalität ${ }^{\text {“32 }}$ gegen eine aktive, kreative Gestaltung der eigenen Erscheinung einzutauschen. Dabei werden Geschlechtsdarstellungen nach Hirschauer von Interaktionspartnern „gelesen“: „Sie bestehen aus sexuierten Darstellungselementen, die ein Betrachter z.T. als ,Geschlechtsmerkmal oder -indiz, aber auch als ,typisch“ männliche/weibliche Eigenschaft oder als ,gehöriges Verhalten“ erkennen kann“. ${ }^{33}$

Ein Problem für Transgenders ist es, ihre empfundene Geschlechtszugehörigkeit ,anderen so zu vermitteln, wie es die Alltagserfahrung ,offensichtlicher "Geschlechtszugehörigkeit erfordert, nämlich nicht verbal“"34 und dabei nicht beim Darstellen ertappt zu werden „im Sinne eines bloßen Spielens oder Vorgebens“. ${ }^{35} \mathrm{Da}$ „,der ihnen zugewachsene Körper [... ] als ,Darstellungs-

27 Hirschauer (1999), S. 26.

28 Hirschauer (1999), S. 32.

29 Hirschauer (1999), S. 37.

30 Hirschauer (1999), S. 45.

31 Hirschauer (1999), S. 38.

32 Ebd.

33 Hirschauer (1999), S. 39.

34 Hirschauer (1999), S. 42.

35 Hirschauer (1999), S. 41. 
material“ Nachteile [bietet]““ ${ }^{36}$ „,arbeiten [sie] zunächst fast ausschließlich gegen ihren Körper: sie bearbeiten ihn durch Make-up, Epilationen, Hormonbehandlungen und kosmetische Operationen, aber auch durch Diät und Bodybuilding“. ${ }^{37}$ Als „situative ,Informationskontrolle““ bezeichnet Hirschauer weitere „Arrangements mit Körperpartien, die als Geschlechtszeichen gelten“ wie das „Kaschieren und prothetische Fingieren“ oder „gestische Konstruktionen von ,sekundären Geschlechtsmerkmalen““.38

Zusätzlich stellt sich die Frage, wie es Transgenders gelingt, eine möglichst mühelose Darstellung zu erreichen. So besteht bezüglich des Maßes der Verwendung von Geschlechtsindizien die Gefahr, „zuviel Aufmerksamkeit zu erzeugen, ohne die nötige Kompetenz zu haben, eine stimmige Erscheinung zu produzieren“. ${ }^{39}$ Dabei wäre es auch wichtig zu überlegen, wie sich das Gefühl beim Darstellen in Richtung einer „Selbstvergessenheit“ entwickeln ließe, die den Darstellern ermöglichen würde, „ihre eigene Darstellung nicht als solche erkennen zu müssen“. ${ }^{40}$ Statt einem mentalen Wissen, was Männer ausmacht, „das bei der Durchführung von Darstellungen eher hinderlich ist“, empfiehlt Hirschauer einen ,,wissenden“ Körper“" ${ }^{41}$

„Als fleischliches Gedächtnis von Darstellungen trägt er wesentlich zu ihrer Mühelosigkeit und damit Unkenntlichkeit als Darstellung bei. Unter dieser Voraussetzung können Darstellungen für einen Betrachter einen geschlechtlichen Körper hervorbringen, als habe er ihnen zugrunde gelegen, und als seien sie nur sein natürlicher ,Verhaltensausdruck ““. ${ }^{2}$

Im Folgenden wird am Beispiel von Transmännern der Frage nachgegangen, welche Möglichkeiten die Betroffenen haben, um ihre Geschlechtsdarstellung in diesem Sinne zu verändern. Dabei wird insbesondere auf die Interaktion von Veränderungen des körperlichen Erscheinungsbildes und stimmlicher Geschlechtsdarstellung eingegangen: ${ }^{43}$ So kann ein - im Sinne der oben beschriebenen Kriterien Hirschauers - stimmiges optisches Erscheinungsbild die Einordnung der Stimme als „männlich“ positiv beeinflussen, ebenso wie eine unmittelbar als männlich wahrgenommene Stimme Uneindeutigkeiten in der übrigen Darstellung überdecken kann.

\section{Veränderung von Geschlechtsdarstellung: Ein unzureichend genutztes Potential?}

Bei Transmännern ist nach Abschluss der Pubertät und vor der Einleitung geschlechtsverändernder Maßnahmen in der Regel von einem chromosomalen Geschlecht sowie von primären und sekundären Geschlechtsmerkmalen einer

\footnotetext{
36 Hirschauer (1999), S. 42.

37 Ebd.

38 Ebd.

39 Hirschauer (1999), S. 43.

40 Hirschauer (1999), S. 47.

41 Hirschauer (1999), S. 48.

42 Ebd.

43 Van Borsel et al. (2000).
} 
biologischen Frau auszugehen. Dabei kann sowohl die Ausprägung der sekundären Geschlechtsmerkmale (wie z. B. Brüste, Hüftrundung, Körperbehaarung, Dimensionen des Stimmorgans) als auch das Ausmaß des Leidensdrucks in Bezug auf die eigene körperliche Ausstattung individuell unterschiedlich sein. Davon hängt ab, welche Anstrengungen ein Transmann unternimmt, um als Mann wahrgenommen zu werden oder sich selber als einen solchen sehen und empfinden zu können. Dementsprechend können die angewendeten Methoden und das Ausmaß der gewünschten Veränderung der Geschlechtsdarstellung individuell verschieden sein; auch streben, wie erwähnt, nicht alle Transmänner eine eindeutige, dauerhaft veränderte Geschlechtsdarstellung in Richtung auf das männliche Geschlecht an. Es gibt Personen, denen eine uneindeutige Darstellung eher entspricht oder die nur zeitweise die eine oder die andere Darstellung wählen wollen.

Bezüglich der Konsequenzen der eingesetzten Methoden für den Körper kann man grundsätzlich zwischen irreversiblen und reversiblen Maßnahmen zur Veränderung der Geschlechtsdarstellung unterscheiden. Zu den irreversiblen Maßnahmen gehören die Verabreichung von gegengeschlechtlichen Hormonen und die Transformationsoperationen. Beide Maßnahmen sind in den meisten ihrer Auswirkungen (im Sinne einer vollständigen Wiederherstellung der ursprünglichen Verhältnisse) nicht mehr rückgängig zu machen und gegebenenfalls mit Nebenwirkungen und z.T. erheblichen Komplikationen verbunden. Reversible Maßnahmen zur Veränderung der Geschlechtsdarstellung sind dementsprechend solche, die nicht mit einer bleibenden Veränderung des Körpers einhergehen. Dabei können Transgender die Tatsache, dass sowohl bestimmte Körperregionen als auch Gegenstände und Verhaltensweisen sexuiert werden können, für sich nutzen. Bei Transmännern, die anstreben, als Männer wahrgenommen zu werden, gibt es demnach zwei Möglichkeiten:

1. das Verdecken von weiblich attribuierten Merkmalen,

2. das Konstruieren von männlich attribuierten Merkmalen.

Für die Erzeugung eines männlichen Erscheinungsbildes gibt es mehrere Ansatzpunkte wie das Tragen von Männerbekleidung, von Herrenfrisuren, von Penisprothesen, das Anbringen von künstlichen Bärten, das Verwenden eines männlichen Vornamens bzw. der Vollzug einer amtlichen Vornamensänderung, das Einüben von „männlicher“ Körperhaltung und Gang oder aber die Maskulinisierung von Stimm- und Sprechverhalten.

Der letztgenannte Punkt soll im Folgenden einer näheren Betrachtung zugeführt werden, da wir ihm eine besondere Relevanz bei der Frage nach der Akzeptanz des Transmannes in seiner gesellschaftlichen Rolle als Mann zuschreiben.

\section{Zur Anpassung von Stimme und Sprechverhalten als akzeptanzsteigernde Maßnahme}

Aus medizinischer Sicht entwickeln sich unter dem Einfluss von Hormonwirkungen während der Pubertät geschlechtsspezifische Stimmeigenschaften, die als eines der sekundären Geschlechtsmerkmale angesehen werden. Das 
biologische Korrelat dieser Stimmveränderungen ist das besondere, hormonell gesteuerte Larynxwachstum der Jugendlichen beider Geschlechter. Skelettal resultiert hierbei nach Kahane (1978) ein anterior-posteriores Ausmaß des männlichen Schildknorpels, das um etwa 20 \% größer ist als das des weiblichen, während die für die Sprechstimmlage relevante membranöse Stimmlippenlänge von Männern und Frauen sogar um 60\% differiert. Am äußeren Hals sichtbar ist die typische Schildknorpelkonfiguration bei Männern, die man als Adamsapfel bezeichnet.

Gegengeschlechtliche Hormongaben bei Transgendern nach Abschluss der Pubertät wirken sich bei Angehörigen des männlichen und weiblichen Ursprungsgeschlechts unterschiedlich aus. Während für Östrogengaben bei Mann-zu-Frau-Transgendern eine stimmanhebende Wirkung nicht nachgewiesen werden konnte, löst eine längerfristige Testosteron- bzw. Anti-Östrogengabe bei Frau-zu-Mann Transgendern offensichtlich zumindest eine partielle Stimmveränderung im Sinne der Adaption an das Zielgeschlecht aus. Die überwiegende Zahl der Untersuchungen zur stimmlichen Situation von Transgenders beschäftigt sich mit Transfrauen (sogenannten Mann-zu-FrauTranssexuellen), da bei dieser Personengruppe besondere stimmanpassende Behandlungsmaßnahmen erforderlich sind, um die Stimme im Sinne einer weiblichen Geschlechtsdarstellung zu verändern. Im Hinblick auf Transmänner wird lediglich in allgemein gehaltenen Veröffentlichungen zur körperlichen Situation von Transsexuellen als Auswirkung der Hormonbehandlung der „Stimmbruch“ erwähnt ${ }^{44}$ - eine Bezeichnung, die auch unter Betroffenen oder in den Aufklärungsgesprächen vor Beginn der Hormonbehandlung üblich ist. Sie impliziert die Annahme eines in den meisten Fällen problemlosen Verlaufs der Stimmveränderungen, die zu Stimmeigenschaften führen, die denen von biologischen Männern entsprechen. Als Begründungen für die fehlende Bezugnahme auf Transmänner in der Stimmliteratur werden neben der angeblich geringeren Prävalenz von Frau-zu-Mann-Transsexualität die (vermeintlich) erfolgreiche Maskulinisierung der Stimme durch die Verabreichung von Testosteron ${ }^{45}$ und der im Vergleich zu Transfrauen weniger problematische Verlauf der angestrebten Stimmveränderungen ${ }^{46}$ genannt. ${ }^{47}$ Tatsächlich haben jedoch Gespräche mit Betroffenen ergeben, dass unter Transmännern eine

44 Z. B. Alter (1999), S. 73; Becker et al. (1997), S. 5; Eicher (1992), S. 88.

45 Vgl. z. B. Oates/Dacakis (1997), S. 185.

46 Vgl. z. B. De Vries/Te Slaa (1986), S. 138; Keil (1994), S. 5; Van Borsel et al. (2000), S. 428; Van Borsel/De Cuypere/ Van den Berghe (2001), S. 570.

47 Zusammenfassend kann aufgrund fehlender systematischer Studien zur stimmlichen Situation von Transmännern bisher keine Aussage darüber getroffen werden, welche anatomisch-morphologischen Veränderungen bei Transmännern nach Verabreichung von synthetischem Testosteron auftreten können und inwiefern sie der natürlichen in der Pubertät von biologisch männlichen Jugendlichen nahe kommen. Das bisherige Wissen basiert wesentlich auf lupenlaryngoskopisch diagnostizierbaren Stimmlippenveränderungen i.S. einer vermehrten Durchblutung und Flüssigkeitseinlagerung. Erkenntnisse vonseiten laryngealer Bildgebung im Verlauf der Hormongabe liegen bisher nur ansatzweise vor. Auch kann bisher nicht prognostiziert werden, in welchem Ausmaß und über welchen Zeitraum es zu individuellen Einschränkungen der Stimmfunktion kommen wird. 
große Unsicherheit besteht, wie mit der besonderen stimmlichen Situation umzugehen ist. ${ }^{48}$

In der phoniatrisch-logopädischen Literatur finden sich Veröffentlichungen zu den so genannten geschlechtsspezifischen Merkmalen von Stimmgebung und Sprechen besonders in Bezug auf drei Grundthemen: 1) Veränderungen der Stimmfunktion während der Mutation (Stimmbruch), 2) Unterschiede der mittleren Sprechstimmlage beider Geschlechter und deren spontansprachlicher Variation (Prosodie), 3) geschlechtsabhängige Eigenschaften in Bezug auf die Lautgestaltung beim Sprechen, wie sie sich in bekannten Unterschieden zur vokaltraktabhängigen Formantgestaltung formulieren läßt.

Dass geschlechtsbezogene Stimmunterschiede nicht ausschließlich biologischen Ursprungs sein können, zeigen Stimmenimitatoren aus dem kabarettistischen und sängerischen Bereich. Manchen von ihnen gelingt es, auch die Stimmeigenschaften, die im Allgemeinen nicht ihrem biologischen Geschlecht zugeschrieben werden, überzeugend nachzuahmen (z. B. Georgette Dee, Malediva etc.) oder mithilfe ihrer Stimme beim Zuhörer Irritationen bzgl. seiner Zuschreibungsgewohnheiten zu erzeugen (z. B. Marla Glen, George Michael, Grace Jones).

Dennoch existieren im Alltagsverständnis Vorstellungen davon, dass sich menschliche Stimmen in Bezug auf Geschlecht voneinander unterscheiden lassen. Oates und Dacakis bezeichnen diese Vorstellungen als „voice stereotypes“" ${ }^{49}$ bzw. „speech stereotypes“. ${ }^{50}$ Die Geschlechtsdarstellung und -wahrnehmung bzw. -zuschreibung von Stimmen ist als ein Konglomerat aus biologischen Faktoren, bevorzugten Stimm- und Sprechverhaltensweisen und spezifischen Vorerwartungen von Zuhörern (die auch das „Alltagswissen von der Zweigeschlechtlichkeit “51 beinhalten) zu verstehen. Deswegen stellt sich die Frage, was Forscher eigentlich jeweils meinen, wenn sie von geschlechtsspezifischen Stimmeigenschaften sprechen. Oates und Dacakis (1997) betonen in diesem Zusammenhang allgemein, dass die beobachtete interindividuelle Variationsbreite bzgl. der untersuchten Eigenschaften des Kommunikationsverhaltens und die Überlappungsbereiche zwischen den Geschlechtern sehr groß sind und bemerken deshalb:

„Voice features are not used exclusively by one gender and instead, should be considered as ,sex preferential' rather than ,sex exclusive“" ${ }^{52}$

48 Aus der Sicht derer, die nicht mit der Hormonbehandlung begonnen hatten, herrschte die Frage vor, wie lange es dauern würde, bis die Stimme endlich tief wäre und ob sich ein Adamsapfel herausbilden würde oder nicht. Diejenigen, die schon länger mit Testosteron behandelt wurden, fragten, wann der Stimmbruch endlich beendet sei, weil sie noch Einschränkungen in der Stabilität der Sprechstimme, ihrer Lautstärke und Belastbarkeit sowie der Singstimme bemerkten, die ihre anfängliche Attraktivität als Anzeichen von „Vermännlichung“ verloren hatten. Von ihnen angesprochene Phoniater und Logopäden hatten in der Regel keine Erfahrung mit dieser seltenen Klientengruppe.

49 Oates/Dacakis (1997), S. 178.

50 Oates/Dacakis (1983), S. 141.

51 Hirschauer (1999), S. 27.

52 Oates/Dacakis (1997), S. 181. 
Damit ist gemeint, dass Menschen sich anhand ihrer Stimmeigenschaften nicht immer eindeutig einer der beiden Geschlechtskategorien zuordnen lassen.

Oates und Dacakis (1997) teilen somit die Auffassung von Wissenschaftlern wie Hirschauer (1999), ${ }^{53}$ die Geschlecht als eine „interaktive Konstruktion“ begreifen. Hiernach käme Stimmen nicht die Qualität eines (zweifelsfreien) Geschlechtsmerkmals zu. Stattdessen betreiben Menschen auf der Basis ihres Stimmorgans und mithilfe bestimmter bevorzugter Verhaltensweisen (z. B. laut oder leise zu sprechen, mit viel oder wenig Variabilität in der Stimmlage etc.) eine stimmliche Selbstdarstellung, die gegebenenfalls auch eine Darstellung ihres Geschlechtszugehörigkeitsempfindens beinhaltet. Ihre Zuhörer nehmen demnach den Stimmklang mit seinen Eigenschaften wahr und schreiben auf der Basis ihrer Vorstellung von Geschlecht und den dazugehörigen Stimmstereotypen den einzelnen Stimmeigenschaften gegebenenfalls eine geschlechtliche Bedeutung zu.

Vor dem Hintergrund dieser Ausführungen erscheint es sinnvoll, sich mit den Stimmeigenschaften per se sowie mit den Einflussfaktoren zu beschäftigen, anstatt sie vorschnell in ein dichotomes Schema einzuordnen. Darüber hinaus haben Transgender, die ihre stimmliche Selbstdarstellung im Rahmen von logopädischer Stimmarbeit verändern wollen, nur mit dieser Sichtweise eine Chance zur aktiven Umgestaltung, denn wäre das falsche Stimmorgan eine „körperliche Fatalität“ ${ }^{54}$ hätte ihre Stimme auch für immer die falschen Merkmale. Vor dem Hintergrund dieser Überlegungen versuchen wir den Schwerpunkt nicht auf die Frage nach den geschlechtsspezifischen Stimmeigenschaften und ihren Normbereichen zu legen, sondern die beobachteten Eigenschaften und ihre Einflussfaktoren zu beschreiben und davon die Zuschreibungspraxis von Geschlecht zu trennen.

\subsection{Stimmgebung, Vokaltraktgestaltung und Geschlechtsdarstellung}

In der Fachliteratur werden die Dimensionen des Stimmorgans im Allgemeinen als die entscheidenden biologischen Einflüsse auf die Geschlechtsdarstellung von Stimmen betrachtet, wobei dieser Begriff sämtliche an der Phonation beteiligte Organstrukturen einschließlich deren nervaler Steuerung umfasst. In Bezug auf die Funktionsweise der laryngealen Komponente des Stimmorgans kommt dem Einfluss des Geschlechtshormons Testosteron im Rahmen der körperlichen Umgestaltung während der biologisch männlichen Pubertät sowie bei synthetischer Verabreichung zur Behandlung bestimmter gynäkologischer Erkrankungen besondere Bedeutung zu. Dagegen sind Auswirkungen auf Brustresonanzeigenschaften und Vokaltraktkonfigurationen primär nicht anzunehmen. Für nähere anatomische und physiologische Aspekte der Stimmgebung sei an dieser Stelle auf klassische Phoniatrielehrbücher verwiesen. ${ }^{55}$ Hier soll lediglich ein sehr knapper Abriss der Zusammen-

53 Hirschauer (1999), S. 25.

54 Hirschauer (1999), S. 38.

55 Z. B. Wendler et al. (1996); Böhme (1997) etc. 
hänge gegeben werden, soweit sie für die nachfolgend beschriebene Stimmarbeit von Belang sind:

Als Stimmorgan werden alle organischen Strukturen bezeichnet, die an der Gestaltung des Stimmklangs beteiligt sind und mit den Teilfunktionen der Stimme (Atmung, Phonation, Artikulation) korrespondieren. Die wichtigsten beteiligten Organe sind für die Atmung die Lunge und die Atemmuskulatur, für die Stimmgebung sind dies physiologischerweise die Stimmlippen, pathologischerweise die Taschenfalten, und für die Artikulation ist dies der Vokaltrakt bzw. das Ansatzrohr, der als Resonator fungierende Hohlraum, der sich zwischen den Stimmlippen und den Lippen erstreckt und sich in Rachen-, Mund- und Nasenraum unterteilen lässt. Durch die Funktion und das Zusammenwirken dieser organischen Strukturen werden die akustischen Merkmale der Stimme gestaltet. Während der Pubertät verändern sich die Dimensionen von Kehlkopf, Stimmlippen und Vokaltrakt. Zusätzlich kommt es dabei zu signifikanten Unterschieden zwischen Angehörigen unterschiedlichen biologischen Geschlechts. So weisen die männlichen Stimmlippen im Vergleich zu den weiblichen im Mittel eine doppelt so große Längenzunahme auf: 10,87 mm gegenüber $4,16 \mathrm{~mm}$, einer resultierenden Stimmlippenlänge von 9 bis $13 \mathrm{~mm}$ bei Frauen und 15 bis $20 \mathrm{~mm}$ bei Männern entsprechend. ${ }^{56}$ Das Kehlkopfwachstum führt neben seinen Auswirkungen auf akustische Merkmale der Stimme gegebenenfalls zu einer optischen Veränderung des vorderen Halsbereichs, die zur Geschlechtsdarstellung beitragen kann und umgangssprachlich als „Adamsapfel“ bezeichnet wird. Gemeint ist die sichtbare Prominenz des Schildknorpels, die ab der Pubertät bei einigen Menschen deutlich in Erscheinung tritt.

Um einschätzen zu können, welche Auswirkungen die Veränderungen der Dimensionen des Stimmorgans während der Pubertät für die jeweiligen akustischen Merkmale der Stimme haben könnten, werden im Folgenden die Ergebnisse von Studien zusammengefasst, die sich mit der Frage nach den Wechselwirkungen zwischen Länge und Masse der Stimmlippen, der Vokaltraktlänge und den Eigenschaften der Stimme, die zur Geschlechtsdarstellung beitragen, beschäftigt haben. Titze (1989) führt den überwiegenden Teil stimmlicher Unterschiede zwischen biologischen Männern und Frauen auf Differenzen in der Länge des frei schwingenden Teils der Stimmlippen zurück. ${ }^{57} \mathrm{Er}$ nimmt weiterhin an, falls die effektive Stimmlippenmasse pro Längeneinheit bei Männern größer sei als die bei Frauen, so sei der Frequenzunterschied der Stimmen im Wesentlichen durch die unterschiedliche Amplitude der Stimmlippenschwingungen bedingt, weniger durch die Unterschiede hinsichtlich der anatomischen Dicke der Stimmlippen.

Fitch und Giedd (1999) konnten im Rahmen ihrer Magnetresonanztomographie-Studie eine positive Korrelation zwischen Vokaltraktlänge, Körpergröße (Länge und Masse) und Lebensalter dokumentieren sowie einen signifikanten Geschlechtsunterschied bzgl. der Vokaltraktlänge ab dem 15. Lebensjahr. Die Stimmlippenlänge selbst scheint dagegen mit dem Halsumfang zu korrelieren (Titze 1989).

56 Kahane (1978), S. 18.

57 Titze (1989), S. 1701. 
In die logopädische Therapie bei intendierter Veränderung der Geschlechtsdarstellung können neben den phonatorischen Verhaltensweisen (Stimme) grundsätzlich verbale und linguistische (z. B. Artikulation, Sprechgeschwindigkeit, Unterbrechungsverhalten, Wortwahl, Gesprächthemen etc.) und nonverbale bzw. non-linguistische (Haltung, Gestik, Mimik etc.) Verhaltensweisen einbezogen werden, da für all diese Bereiche mehr oder weniger sorgfältig untersuchte Geschlechtsunterschiede bestehen sollen, ${ }^{58}$ die bei erfolgreicher Imitation gegebenenfalls die gewünschte Geschlechtswahrnehmung und -zuschreibung beim Gegenüber bewirken können.

Die Grundfrequenz der Stimmlippenschwingung und ihr perzeptives Korrelat, die mittlere Sprechstimmlage, ist die im Zusammenhang mit der Kategorie Geschlecht am häufigsten erwähnte Stimmeigenschaft. In der Regel beziehen sich Menschen auf dieses Merkmal, wenn man sie fragt, wie sich die Stimmen von Frauen und Männern unterscheiden. So haben Untersuchungen ergeben ${ }^{59}$ dass folgende Stereotypen vorherrschen: Frauen haben hohe Stimmen und Männer haben tiefe Stimmen. Perzeptionsstudien mit Transfrauen zeigten, was die meisten Menschen unter hoch bzw. tief verstehen:

„Fundamental frequency was highly correlated with speaker sex and [...] transsexuals who used a fundamental frequency of $160 \mathrm{~Hz}$ or more were likely to be judged as female. Those with a fundamental frequency below $160 \mathrm{~Hz}$ were perceived to be male“. ${ }^{60}$

Andere Autoren beschreiben den Bereich zwischen $145 \mathrm{~Hz}$ und $165 \mathrm{~Hz}$ als geschlechtsindifferenten Bereich. Betrachtet man die Lage des sogenannten Normstimmfelds bzgl. des Singstimmumfangs von Erwachsenen, also den Bereich, innerhalb dessen ein Mensch die Grundfrequenz seiner Stimmlippenschwingung leise und laut variieren kann, gilt für Frauen ein Bereich von 147 bis $784 \mathrm{~Hz}$ und für Männer von 87 bis $587 \mathrm{~Hz} .{ }^{61}$ Hierbei ist anzumerken, dass die entspannte Stimmgebung der Sprechstimme, die so genannte Indifferenzlage üblicherweise wenige Halbtöne (Terz-Quinte) oberhalb der unteren Stimmumfangsgrenze des Erwachsenen liegt. Der Bereich um $160 \mathrm{~Hz}$ ist zwar beiden Geschlechtern prinzipiell verfügbar, die Bedingungen seiner Erzeugung weisen aber wesentliche geschlechtsabhängige Unterschiede auf. Ein Blick auf die Normbereiche der Sprechstimmlage ${ }^{62}$ für erwachsene Frauen (Mittelwert: 196 bis $224 \mathrm{~Hz}$, Range: 145 bis $275 \mathrm{~Hz}$ ) und Männer (Mittelwert: 107 bis $132 \mathrm{~Hz}$, Range: 80 bis $165 \mathrm{~Hz}$ ), die im Mittel um eine Oktave differieren, zeigt weiterhin, dass der Überlappungsbereich biologischer männlicher und weiblicher Sprecher mit 145 bis $165 \mathrm{~Hz}$ recht klein ist. Der unangestrengte Wechsel in die Sprechstimmlage des Zielgeschlechtes ist gegebenenfalls nur unter Zuhilfenahme von geeigneten stimmtherapeutischen Verfahren dauerhaft und mit der erforderlichen „Natürlichkeit“ und kommunikativen Variationsbreite möglich.

\footnotetext{
$58 \mathrm{Vgl.} \mathrm{für} \mathrm{einen} \mathrm{Überblick:} \mathrm{Oates/Dacakis} \mathrm{(1983).}$

59 Vgl. Oates/Dacakis (1997), S. 180.

60 Ebd.

61 Schultz-Coulon/Asche (1988).

62 Oates/Dacakis (1997), S. 178.
} 
Perzeptionsstudien, die von einem binären Geschlechtermodell ausgehen, haben ergeben, dass die Sprechmelodien von Frauen im Allgemeinen als „expressiv“ und „melodiös“ eingeschätzt werden, während die von Männern demgegenüber als „monoton“ wahrgenommen werden. ${ }^{63}$ Diese Hörwahrnehmung bezieht sich auf den Tonhöhenverlauf von gesprochener Sprache in Form von Grundfrequenzschwankungen. Auch in Kenntnis der Längen- und Massenunterschiede männlicher und weiblicher Stimmlippen scheint hier nicht das organische Korrelat, sondern eher ein erworbenes Verhaltensmuster von Bedeutung zu sein.

Die Grundfrequenz der Stimmlippenschwingung und die Formantfrequenzen sind die Parameter der Geschlechtsdarstellung von Stimmen, die am besten untersucht sind und deren gegenüber anderen Stimmmerkmalen überragende Relevanz bezüglich der Geschlechtszuschreibung durch Hörer anhand verschiedener Studien nachgewiesen wurde. ${ }^{64}$ Uneinigkeit besteht lediglich in der Bewertung, welcher dieser beiden Parameter ein Hörerurteil stärker in Richtung des einen oder anderen Geschlechts beeinflusst.

Baken und Orlikoff ${ }^{65}$ sowie Sundberg ${ }^{66}$ geben als eine Zusammenfassung verschiedener Studien aus verschiedenen Gründen vorsichtig anzuwendende Regeln für den Zusammenhang zwischen zu erwartenden Formantfrequenzen und Veränderungen in den Dimensionen und der Gestalt des Vokaltrakts an. So kann eine Verlängerung des Vokaltrakts einerseits durch ein Absenken des Kehlkopfes im Hals und andererseits durch eine verstärkte Lippenrundung bewirkt werden, wodurch alle Formantfrequenzen abgesenkt werden. Eine Reduktion der Kieferöffnung führt zu einer Absenkung des ersten Formanten. Der zweite Formant wird vor allem durch die Zungenposition beeinflusst: Wird die Zungenspitze angehoben und damit der vordere Mundraum verengt, erhöht sich der zweite Formant, wohingegen ein Anheben des Zungenrückens und damit eine Verengung des hinteren Mundraums zu einer Absenkung des zweiten Formanten führt. Der dritte Formant tendiert zu niedrigeren Frequenzwerten, wenn die Zungenspitze am oberen Gaumen anliegt und sich dabei in möglichst großer Entfernung von den Hinterflächen der Schneidezähne befindet. Die höheren Formanten sind viel weniger durch die Artikulation beeinflussbar, sondern werden vor allem durch die konstitutionelle Vokaltraktkonfiguration determiniert.

Darüber hinaus wurden noch andere Stimmparameter bei Männern und Frauen getrennt untersucht und mehr oder weniger replizierbare Unterschiede festgestellt. So wurden für Männer messtechnisch eine Tendenz zu fallenden und weniger variablen Intonationsmustern, eine höhere habituelle Sprechstimmstärke sowie eine rauere Stimmklangqualität ermittelt. ${ }^{67}$ Die entsprechenden perzeptiven Korrelate „monotones, lautes, kraftvolles Sprechen“, fanden sich auch in Erhebungen zu Stereotypen männlichen Sprech- und Stimmverhaltens wieder. ${ }^{68}$ Im Gegensatz zu Grundfrequenz und Formanten

63 Oates/Dacakis (1997), S. 179.

64 Für Literaturhinweise siehe: Oates/Dacakis (1997), S. 179.

65 Baken/Orlikoff (2000), S. 265.

66 Sundberg (1987), S. 22.

67 Oates/Dacakis (1997).

68 Ebd. 
lassen sich bzgl. der letztgenannten Parameter nur schwer organische Korrelate finden, die als entscheidende limitierende oder fördernde Faktoren für die Ausprägung dieser Stimmeigenschaften anzusehen sind.

Da, wie King et al. ${ }^{69}$ berichten, die Verknöcherung der Kehlkopfknorpel erst mit dem 65. Lebensjahr abgeschlossen sein soll, und die Wachstumsprozesse des Stimmorgans ,a product of the combination effect of growth hormone at puberty and testosterone ${ }^{\text {"70 }}$ seien, ist es denkbar, dass auch eine postpubertäre Testosterongabe („pharmacologic puberty “"71) Veränderungen in den Dimensionen von Kehlkopf und Vokaltrakt bewirken könnte. Eher unwahrscheinlich ist dagegen ein Einfluss auf die geometrische Grundstruktur des im $90^{\circ}$-Winkel ausgebildeten männlichen gegenüber dem im $120^{\circ}$-Winkel gestalteten weiblichen Thyroid. Aufgrund des fehlenden Wachstumshormons würde auch die endolaryngeale Hormonwirkung geringer ausfallen, als dies im Rahmen der biologisch männlichen Pubertät („physiologic puberty “72) der Fall wäre. Aufgrund dieser Einschränkung und der von Kahane (1978) nachgewiesenen Tatsache, dass das Längenwachstum der Stimmlippen mit der Pubertät abgeschlossen ist, ist bei einer „pharmakologischen Pubertät“ eine Massenzunahme der Stimmlippen bei gering ausgeprägtem oder fehlendem Längenwachstum anzunehmen . ${ }^{73}$ Im Rahmen einer gängigen nichtinvasiven phoniatrischen Untersuchung ist es allerdings bisher nicht möglich, die Veränderungen am Stimmorgan biologischer Frauen infolge einer Verabreichung androgenhaltiger Substanzen exakt zu dokumentieren, da bisher nur endoskopische Verlaufsbeurteilungen vorliegen. Das bedeutet auch, dass keine gesicherten Annahmen für Art und Ausmaß der Veränderungen des Stimmorgans von Transmännern existieren, zumal die Übertragbarkeit der Untersuchungsergebnisse der biologischen Frauen mit iatrogenen Stimmstörungen nach Gabe von androgenen oder anabolen Hormonen aufgrund der Unterschiedlichkeit der verwendeten Präparate sowie der Dauer und Dosierung der Hormongabe eingeschränkt ist. Tendenziell scheint es aber so zu sein, dass bei Transmännern als hauptsächliche Veränderung im Stimmorgan infolge der Hormonbehandlung primär von einer Mehrdurchblutung und vermehrten Flüssigkeitseinlagerung in den Stimmlippen auszugehen ist.

Eine Einschätzung der stimmlichen Situation von Transmännern erweist sich angesichts dieser Datenlage als schwierig: Nach dem Modell der Mutation bei biologisch männlichen Jugendlichen wäre die Testosteronbehandlung in Bezug auf eine Veränderung der Geschlechtsdarstellung der Stimme erfolgreich und im Hinblick auf die Funktionsfähigkeit insgesamt unproblematisch. Nach dem Modell der hormonellen Dysphonie bei biologischen Frauen wäre eine veränderte Geschlechtsdarstellung im Sinne einer Übereinstimmung mit den Normbereichen für biologische Männer möglich, aber keinesfalls garantiert. Die Gefahr einer irreversibel eingeschränkten Funktionsfähigkeit bis hin zu einer Berufsunfähigkeit für Sprech- und Stimmberufler wäre deutlich gegeben.

Vorläufige Untersuchungen an Frau-zu-Mann-Transgendern haben ergeben, dass sich die Sprechstimmlage in Abhängigkeit von der Dauer der laufen-

\footnotetext{
69 King et al. (2001), S. 555.

70 King et al. (2001), S. 554.

71 King et al. (2001), S. 555.

72 Ebd.

73 Ebd.
} 
den Hormontherapie verändert, wobei es im ersten Jahr der Einnahme offensichtlich zur deutlichsten Absenkung der Sprechstimmlage und im weiteren Verlauf zu einer zunehmenden Intonationsstabilität der Stimme kommt. ${ }^{74}$

Zusammenfassend ergibt sich aus diesem Vergleich folgende Grundannahme für die stimmliche Situation von Transmännern: Die organischen und funktionellen Voraussetzungen der Stimmgebung von Transmännern während der Behandlung mit Testosteron sind weder mit denen von biologisch männlichen Jugendlichen während oder nach der Mutation noch mit denen von biologischen Frauen bei hormoneller Dysphonie aufgrund der Verabreichung von androgenhaltigen Medikamenten exakt vergleichbar.

Neben der Hormonbehandlung gibt es weitere spezifische Einflussfaktoren auf die Stimmgebung von Transmännern, die sich aus den individuell eingesetzten Maßnahmen zur Veränderung der Geschlechtsdarstellung ergeben. Dabei sind das Ausmaß des Leidensdrucks und dementsprechend die Anstrengungen, die unternommen werden, um die Geschlechtsdarstellung als Mann zu verbessern, von entscheidender Bedeutung.

Darüber hinaus wird davon ausgegangen, dass sich auf die Stimmgebung nicht nur willkürliche Veränderungen des Stimm- und Sprechverhaltens auswirken, sondern auch jegliche Maßnahmen, die die anderen Diagnostikbereiche der logopädischen Stimmarbeit beeinflussen.

Dabei werden dem Kaschieren der Brust durch Abbinden oder eine gebeugte Körperhaltung negative Auswirkungen auf Körperspannung, Phonationsatmung, und Stimmgebung zugeschrieben. Die genannten Methoden zum Verbergen der Brust führen zu einer Fixierung der Haltung des Oberkörpers und damit zu einer Einschränkung der Beweglichkeit und der flexiblen Anpassung der Körperspannung. Dadurch wird auch der Atemraum eingeengt. Der sichtbaren Körperhaltung und -spannung werden darüber hinaus Zusammenhänge zur inneren Haltung eines Menschen zugeschrieben, ${ }^{75}$ wobei einer fixierten Körperspannung und einer gebeugten Crundhaltung ungünstige Auswirkungen auf die Selbstdarstellung eines Menschen zugeschrieben werden. Dem entgegen stehen Ansichten, ein erfolgreiches Abbinden der Brust könne dazu führen, dass eine aufrechte Körperhaltung einzunehmen, wodurch die Selbstdarstellung gegebenenfalls positiv beeinflusst würde. Entsprechend würde die Mastektomie beim Frau-zu-Mann-Transgender eine Entlastung im angegebenen Sinne bewirken, da die Brust nicht länger kaschiert werden muss.

Eine Veränderung des optischen Erscheinungsbilds im Sinne eines besseren Passings lässt positive Auswirkungen auf Körperspannung und Körperhaltung (gesamtkörperliche Entspannung), psychosoziale Situation (bessere Akzeptanz), Selbstdarstellung und damit auch der Phonation erwarten.

Willkürliche Versuche einer Maskulinisierung des Stimm- und Sprechverhaltens könnten, insofern sie die Qualität eines „Stimmmissbrauchs“ annehmen, vor allem auf die Spannungsverhältnisse im Kehlkopf, die Artikulation und die Selbstdarstellung (bei unglaubwürdiger Veränderung im Sinne einer „Verstellung“ der Stimme) negativen Einfluss haben. Im Einzelnen sind hierunter eine Absenkung der Grundfrequenz und der Formanten über eine nach

74 Vgl. Scheidt (2003), S. 95-100.

$75 \mathrm{Vgl}$. Stengel/Strauch (1996), S. 78. 
unten gedrückte Stimme bei enger Kieferweite, knödelnder Rückverlagerung der Zunge und unphysiologischem Einsatz der laryngealen Taschenfalten zu verstehen. Habituell werden prosodische Einschränkungen der Sprechmelodie sowie willkürliche Veränderungen der stimmlichen Klangqualität z. B. über Nikotinabusus und vermehrten Alkoholkonsum stimmschädigend zur Stimmadaption eingesetzt.

Eine zusätzliche Beanspruchung physischen und akustischen Raumes (wie sie biologischen Männern oft zugeschrieben wird) hätte ungünstige Auswirkung auf eine überzeugende Selbstdarstellung und die Phonation, falls sie in einem Ausmaß betrieben würde, das die Funktionsfähigkeit des individuellen Stimmorgans überstrapazierte oder das von Kommunikationspartnern als unangenehm oder unangemessen wahrgenommen würde.

\subsection{Die Stimmarbeit mit Transmännern unter Einbeziehung von Intention und Selbstdarstellung: Grundsätzliche Überlegungen und konkrete Vorschläge}

Diagnostikkonzepte für die logopädische Stimmarbeit leiten sich aus Modellen $\mathrm{ab}$, welche die menschliche Stimme als ein multidimensionales Phänomen ansehen. So stellt Spiecker-Henke ${ }^{76}$ ihre Konzeption der Stimmfunktion folgendermaßen dar:

„Stimme ist das Ergebnis eines hochkomplexen Zusammenspiels von zerebralen Aktivitäten, subkortikalen Regelkreisen, emotionalen Komponenten sowie muskulären Leistungen einschließlich der Respiration, Phonation, Artikulation und der aufgerichteten Körperhaltung."

Aus Sicht der „funktionalen Stimmarbeit““77 ist die Stimmfunktion „vom Ganzen des Individuums, vom Tonus der Nerven und Muskeln, von seiner Körperhaltung und seinen Bewegungen, von seiner psychischen Verfassung und seinen emotionalen Gewohnheiten abhängig“ “ ${ }^{78}$ Als Diagnostikkonzept empfiehlt Spiecker-Henke ein „ganzheitliches Vorgehen“, das „die Gesamtpersönlichkeit des Stimmkranken [hier: des Probanden] in ihrem vielfältigen wechselseitigen Zusammenspiel organischer, psychisch-emotionaler und kommunikativ-sozialer Strukturen sieht" ${ }^{79}$

Aus diesen Beschreibungen ergeben sich die übergeordneten Diagnostikbereiche Körperspannung/Körperhaltung, Atmung, Phonation, Artikulation und psycho-soziale Situation. Für die Untersuchung der stimmlichen Situation von Transmännern können diese Bereiche der allgemeinen logopädischen Stimmdiagnostik übernommen werden. Im Hinblick auf die Besonderheiten der Lebenssituation von Transmännern und den daraus resultierenden speziellen Einflussfaktoren auf die Stimme sind jedoch Ergänzungen der herkömmlichen Untersuchungsbereiche notwendig.

\footnotetext{
76 Spiecker-Henke (1997), S. 6.

77 Rabine/Jacoby (1991).

78 Rabine/Jacoby (1991), S. 4.

79 Spiecker-Henke (1997), S. 6.
} 
So sollte im Bereich Körperspannung/Körperhaltung zusätzlich die Frage nach der Beziehung zum eigenen Körper berücksichtigt werden, um zu untersuchen, inwiefern auf den einzelnen Klienten die viel zitierte Definition Eichers: „Als transsexuell werden Menschen bezeichnet, die sich im falschen Körper wähnen" ${ }^{\text {"8o }}$ zutrifft und welche individuelle Bedeutung diese besondere Art des Körperbezugs für den Umgang mit ihm hat.

Der Diagnostikbereich Phonation, der üblicherweise eine Untersuchung der Stimmfunktion nach Kriterien der Funktionsfähigkeit umfasst, wäre um eine Untersuchung von Parametern der Geschlechtsdarstellung der Stimmen zu erweitern. Da die individuell ergriffenen Maßnahmen zur Veränderung der Geschlechtsdarstellung Auswirkungen auf alle Diagnostikbereiche haben können, sollte in jedem Bereich untersucht werden, inwiefern beobachtete Einschränkungen auf diese Maßnahmen zurückzuführen sind.

Manche stimmtherapeutischen Schulen ${ }^{81}$ untersuchen über die genannten Diagnostikbereiche hinaus die so genannte „Intention“, die das gesamte Kommunikationsverhalten eines Menschen beeinflusst:

„Damit wird die Ausdrucksabsicht und die Ausdrucksfähigkeit eines Menschen bezeichnet, die von seiner Persönlichkeit und seiner Lebensgeschichte geprägt ist. Art und Ausmaß der Intention bei der Kommunikation bestimmen den Muskeltonus eines Menschen und damit Atmung, Phonation und Artikulation. Die Intention zeigt sich [...] in den nonverbalen Ausdrucksfähigkeiten eines Menschen. ${ }^{\text {“82 }}$

In Bezug auf Transmänner sollte zusätzlich untersucht werden, was der einzelne Klient unternimmt, um seine individuelle Verortung in Bezug auf die beiden Geschlechtspole zum Ausdruck zu bringen, wie viel Anstrengung ihn das kostet und inwiefern er dabei erfolgreich ist. Zusammenfassend wird dieser Diagnostikbereich im Folgenden „Selbstdarstellung“ genannt. Diese Bezeichnung impliziert die Untersuchung aller „körperliche[n] Anschauungsbilder“, die die Klienten mehr oder weniger kontrolliert von sich selbst ${ }^{84}$ abgeben . Dabei werden Selbstdarstellungen so verstanden, dass in ihnen die persönliche Wirklichkeit des Darstellenden von seinen Interaktionspartnern „gelesen“ wird. ${ }^{85}$ Im Gegensatz zur Bezeichnung Intention wird mit dem Begriff der Selbstdarstellung das Verständnis einer „interaktiven Konstruktion“ von persönlicher Wirklichkeit betont. Eine gelungene Selbstdarstellung läge dementsprechend dann vor, wenn der Klient, die Art und Weise, wie er die ihm wichtigsten persönlichen Eigenschaften zum Ausdruck bringt, persönlich als stimmig erlebt und mit der Wirkung auf andere zufrieden ist.

Aus den bisherigen Ausführungen ergibt sich die Notwendigkeit eines Diagnostikkonzepts, das insbesondere alle relevanten stimmlichen Parameter im Hinblick auf Geschlechtsdarstellung und Funktionsfähigkeit sowie ihre Einflussfaktoren entwickelt und erprobt. Im Einzelnen sollten die erhobenen Parameter Antworten auf folgende Fragestellungen ermöglichen:

80 Eicher (1992), S. 1.

$81 \mathrm{Vgl}$. Coblenzer/Muhar (1993).

82 Daniel/Kellner (1990), S. 23.

83 Hirschauer (1999), S. 39.

$84 \mathrm{Vgl}$. Stengel/Strauch (1996), S. 20.

85 Hirschauer (1999), S. 39. 
1. Welche speziellen Einflussfaktoren auf Stimme gibt es bei den untersuchten Transmännern?

2. Wie nehmen die Probanden ihre stimmliche Situation wahr und welche Wünsche haben sie an ihre Stimme?

3. Welche Hinweise auf behandlungsbedürftige Auffälligkeiten zeigen sich in Bezug auf die stimmliche Geschlechtsdarstellung und Funktionsfähigkeit: a) aus phoniatrischer Sicht?, b) aus logopädischer Sicht?, c) aus der Sicht von Fremdbeurteilern?

4. Welche Unterschiede in den Stimmeigenschaften ergibt der Vergleich von: a) Transmännern und biologischen Frauen bzgl. der Geschlechtsdarstellung, b) Transmännern mit einer Dauer der Hormonbehandlung $<1$ Jahr versus > 1 Jahr bzgl. der Geschlechtsdarstellung und der Funktionsfähigkeit?

5. Welche Schlussfolgerungen lassen sich in Bezug auf ein geeignetes Diagnostikkonzept für die logopädische Stimmarbeit ziehen?

Das von der Klinik für Phoniatrie in Aachen auf der Grundlage dieser Überlegungen entwickelte Konzept beinhaltet im Einzelnen (1) eine phoniatrische Untersuchung des Stimmorgans, (2) ein semistandardisiertes Interview (logopädisches Gespräch), (3) eine akustische Analyse von Aufnahmen der Sprech-, Singund Kraftstimme sowie (4) eine Perzeptionsstudie zur Erfassung der Fremdwahrnehmung der Geschlechtsdarstellung der Stimmen (vgl. Abbildung 1). ${ }^{86}$

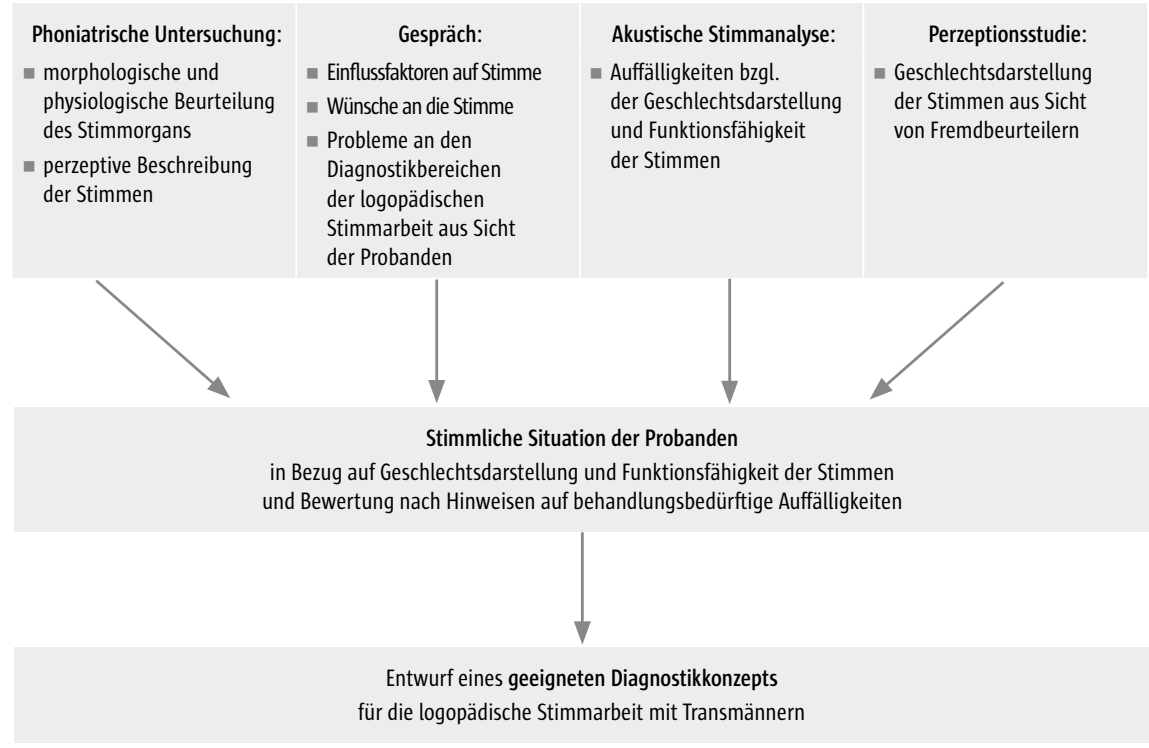

Abb. 1 Perzeptionsstudie zur Erfassung der Fremdwahrnehmung der Geschlechtsdarstellung der Stimmen

86 Zu den durchgeführten Untersuchungen vgl. Scheidt (2003). 
Die phoniatrische Untersuchung dient einer Beurteilung der Morphologie und Physiologie des Stimmorgans sowie einer perzeptiven Bewertung der Stimmen. ${ }^{87}$

Im logopädischen Gespräch werden die individuellen Einflussfaktoren auf die Stimmgebung, die Wünsche der Probanden an ihre Stimme und ihre Selbstwahrnehmung von ihren Problemen in den Diagnostikbereichen der logopädischen Stimmarbeit erfragt. Anhand der akustischen Stimmanalyse werden messtechnisch Auffälligkeiten der Geschlechtsdarstellung und Funktionsfähigkeit der Stimmen ermittelt. Im Rahmen der Perzeptionsstudie soll schließlich die Fremdwahrnehmung der Stimmen in Bezug auf die Kategorie Geschlecht kontrolliert untersucht werden. Das logopädische Gespräch wird gegenüber herkömmlichen Anamnesegesprächen zur logopädischen Stimmarbeit ${ }^{88} \mathrm{um}$ folgende Inhalte erweitert: Verortung des individuellen Probanden in Bezug auf die Geschlechtspole männlich und weiblich (empfundene Geschlechtszugehörigkeit), Maßnahmen zur Veränderung der Geschlechtsdarstellung, erlebte Stimmveränderungen infolge der Hormonbehandlung, Selbstwahrnehmung der eigenen Stimme in Bezug auf die Kategorie Geschlecht, Geschlechtszuschreibung im Alltag sowie psychosoziale Situation in Hinblick auf die gelebte Transsexualität. Die Ergebnisse dieser Erhebung der subjektiven Einschätzung ihrer stimmlichen Situation durch die Probanden werden durch objektive Untersuchungsverfahren ergänzt. ${ }^{89}$

Eine gemeinsame Betrachtung der Ergebnisse aus allen Untersuchungsteilen ergibt eine Einschätzung der stimmlichen Situation der Probanden in Bezug auf die Geschlechtsdarstellung und die Funktionsfähigkeit ihrer Stimmen und offenbart so konkrete Möglichkeiten einer aktiven Gestaltung der Kategorie Geschlecht durch die Betroffenen.

\section{Zusammenfassung}

Mit Blick auf das Phänomen Transsexualität kommt der Medizin die Rolle einer Deutungs- oder Definitionsmacht zu: Sie klassifiziert Transsexualität als Störung der Geschlechtsidentität und schreibt ihr folglich einen Krankheitscharakter zu. Diese „Pathologisierung“ ermöglicht den Betroffenen einerseits die Inanspruchnahme bestimmter medizinischer Leistungen. Andererseits wurde auf diese Weise ein Krankheitsbild geschaffen, das am herkömmlichen Prinzip der Zweigeschlechtlichkeit festhält. Eben dies stimmt jedoch nicht mit dem Selbstbild vieler Betroffener überein: ein Teil der Transgender fühlt sich weder als Frau noch als Mann oder sowohl weiblich als auch männlich und leidet deswegen unter der in der ICD-10 vorgenommenen einseitigen Zuschreibung zu einem Geschlecht. Wer "Geschlecht“ jedoch als soziale Konstruktion begreift,

87 Zu berücksichtigen ist ferner, wie die Stimmen der Probanden im Hinblick auf eine Geschlechtszuschreibung von Kommunikationspartnern wahrgenommen werden. Die Fremdwahrnehmung im Alltag (am Telefon und bei Begegnungen) in Bezug auf die Kategorie Geschlecht wird daher einerseits im Gespräch erfragt, andererseits ergibt die phoniatrische Untersuchung Ergebnisse zur Geschlechtszuschreibung aufgrund des perzeptiven Eindrucks von der Sprechstimme.

88 Vgl. z. B. Sataloff (1997), S. $193 \mathrm{ff}$.

89 Zu den durchgeführten Untersuchungen vgl. Scheidt (2003). 
versteht auch die Geschlechtsdarstellung und -zuschreibung von Menschen als ein Verhalten, das aktiv gestaltet bzw. beeinflusst werden kann. Dies trifft auch auf die Stimme zu, der in der Regel eine sehr wichtige Rolle in der Geschlechtsdarstellung eines Menschen zugeschrieben wird, so dass Transgender der Anpassung ihrer Stimme besondere Bedeutung beimessen.

Vor dem Hintergrund dieser Annahmen haben Betroffene, die ihre stimmliche Selbstdarstellung im Rahmen von logopädischer Stimmarbeit verändern wollen, unseres Erachtens eine besondere Chance, auf ihre Geschlechtsdarstellung unmittelbar Einfluss zu nehmen und so gegebenenfalls die gesellschaftliche Akzeptanz ihrer Selbstdarstellung zu erhöhen.

Im vorliegenden Beitrag wurde daher der Versuch unternommen, dieses Konzept für den Bereich der Stimmarbeit zu explizieren und das Potential einer solchen Vorgehensweise auszuleuchten.

\section{Literatur}

Alter (2005): Helma Katrin Alter, Gleiche Chancen für alle. Transidentität in Deutschland 1998/1999, Norderstedt 2005

Baken/Orlikoff (2000): Ronald J. Baken, Robert F. Orlikoff, Clinical Measurement of Speech and Voice, San Diego 2000

Becker et al. (1997): Sophinette Becker, Hartmut A. G. Bosinski, Ulrich Clement et al., Standards der Behandlung und Begutachtung von Transsexuellen der Deutschen Gesellschaft für Sexualforschung, der Akademie für Sexualmedizin und der Gesellschaft für Sexualwissenschaft, Zeitschrift für Sexualforschung 10 (1997), 2, S. 147-156, www.info.sexualpaedagogik.de/standards.html [14.10.2006]

Böhme (1997): Gerhard Böhme, Sprach-, Sprech-, Stimm- und Schluckstörungen. Ein Lehrbuch, Stuttgart 1997

Clement/Senf (1996): Ulrich Clement, Wolfgang Senf, Transsexualität. Behandlung und Begutachtung, Stuttgart 1996

Coblenzer/Muhar (1993): Horst Coblenzer, Franz Muhar, Atem und Stimme, Wien 1993

Daniel/Kellner (1990): Susanne Daniel, Marita Kellner, Logopädische Diagnostik und Therapie bei Stimmstörungen. Unveröffentlichtes Skript der Staatlich anerkannten Lehranstalt für Logopäden der medizinischen Einrichtungen der RWTH Aachen, Aachen 1990

De Vries/te Slaa (1986): C. de Vries, M. te Slaa, Logopedische therapie bij transsexuelen, Logopedie en Foniatrie 58 (1986), p. $138 \mathrm{f}$.

Eicher (1992): Wolf Eicher, Transsexualismus. Möglichkeiten und Grenzen der Geschlechtsumwandlung, Stuttgart 1992

Fitch/Giedd (1999): W. Tecumseh Fitch, Jay Giedd, Morphology and development of the human vocal tract. A study using magnetic resonance imaging, Journal of the Acoustical Society of America, 106 (1999), 3, p. 1511-1522

Groß/Steinmetzer (2007): Dominik Groß, Jan Steinmetzer, Transsexualität zwischen Medizin, Recht und Ethik. Ein europäischer Vergleich, in: B. Sharon Byrd, Jan C. Joerden, Jahrbuch für Recht und Ethik, Bd. 14, 2006/07, S. 581-609

Hirschauer (1999): Stefan Hirschauer, Die soziale Konstruktion der Transsexualität, Frankfurt a. M. 1999

Huber (1999): Gerd Huber, Psychiatrie. Lehrbuch für Studium und Weiterbildung, Stuttgart 1999

Jagose (2001): Annamarie Jagose, Queer theory. Eine Einführung, Berlin 2001

Kahane (1978): Joel Kahane, A morphological study of the human prepubertal and pubertal larynx, American Journal of Anatomy 151 (1978), p. 11-20

Keil (1994): Tobias Keil, Transsexualität und Stimme, Sprechen. Zeitschrift für Sprachwissenschaft 12 (1994), 2, S. 4-14 
Kessler/McKenna (2000): Suzanne Kessler, Wendy McKenna, Who put the „Trans“ in Transgender? Gender Theory and Everyday Life, The International Journal of Transgenderism 4 (2000), 3, www.symposion.com/ iit/gilbert/kessler.htm [14.05.2007]

King et al. (2001): Austin King, Jon Ashby, Charles Nelson, Effects of Testosterone Replacement on a Male Professional Singer, Journal of Voice 15 (2001), 4, p. 553-557

Kroll (2002): Renate Kroll (Hrsg.), Metzler Lexikon Gender Studies Geschlechterforschung. Ansätze - Personen Grundbegriffe, Stuttgart 2002

Migeon/Wisniewski (1998): Claude J. Migeon, Amy B. Wisniewski, Sexual Differentiation. From Genes to Gender, Hormone Research 50 (1998), p. 245-251

Oates/Dacakis (1983): Jenni Oates, Giorgia Dacakis, Speech Pathology Considerations in the Management of Transsexualism. A Review, British Journal of Disorders of Communication 18 (1983), 3, p. 139-151

Oates/Dacakis (1997): Jenni Oates, Giorgia Dacakis, Voice Change in Transsexuals, Venereology 10 (1997), 3, p. $178-187$

Rabine/Jacoby (1991): Eugen Rabine, P. Jacoby, Die drei Teilfunktionen der Stimmfunktion, in: Walter Rohmert (Hrsg.), Grundzüge des funktionalen Stimmtrainings, Köln 1991, S. 1-56

Roche Lexikon Medizin (1987): Hoffmann-LaRoche AG (Hrsg.), Roche-Lexikon Medizin, München, 2. Aufl., 1987

Sataloff (1997): Robert T. Sataloff, Professional Voice. The Science and Art of Clinical Care, San Diego 1997

Scheidt (2003): David Scheidt, Entwicklung und Erprobung eines Diagnostikkonzeptes für die logopädische Stimmarbeit mit Transgenders am Beispiel der sogenannten Frau-zu-Mann-Transsexualität. Diplomarbeit im Studiengang Lehr- und Forschungslogopädie Aachen 2003 (unveröffentlicht)

Schulte-Fischedick (2002): Valeria Schulte-Fischedick, ,... wie exotische Schmetterlinge an ihren Wänden“. Ein Interview mit Del LaGrace Volcano, in: polymorph (Hrsg.), (K)ein Geschlecht oder viele? Transgender in politischer Perspektive, Berlin 2002, S. 13-30

Schultz-Coulon/Asche (1988): Hans-Jürgen Schultz-Coulon, Sylvia Asche, Das „Normstimmfeld“. Ein Vorschlag, Sprache - Stimme - Gehör 12 (1988), S. 5-8

Sigusch (1997): Volker Sigusch, Transsexualismus. Forschungsstand und klinische Praxis, Nervenarzt 68 (1997), S. 870-877

Sigusch (2005): Volker Sigusch, Praktische Sexualmedizin. Eine Einführung, Köln 2005

Spiecker-Henke (1997): Marianne Spiecker-Henke, Leitlinien der Stimmtherapie, Stuttgart 1997

Steinmetzer/Groß (2006): Jan Steinmetzer, Dominik Groß, Die Behandlung von Transsexualität und ihre ethischen Implikationen. Projektskizze, in: Dominik Groß, Tobias Heinrich Duncker (Hrsg.), Farbe - Erkenntnis - Wissenschaft, Münster 2006 (= Anthropina, 1), S. 177-188

Steinmetzer/Groß/Duncker (2007): Jan Steinmetzer, Dominik Groß, Tobias Heinrich Duncker, Ethische Fragen im Umgang mit transidenten Personen. Limitierende Faktoren des gegenwärtigen Konzepts von „Transsexualität", Ethik in der Medizin 19 (2007), 1, S. 39-54

Stengel/Strauch (1996): Ingeborg Stengel, Theo Strauch, Stimme und Person, Stuttgart 1996

Sundberg (1987): Johan Sundberg, The Science of the singing Voice, Dekalb 1987

Titze (1989): Ingo R. Titze, Physiologic and acoustic differences between male and female voices, Journal of the Acoustical Society of America 85 (1989), 4, p. 1699-1707

Van Borsel et al. (2000): John van Borsel, Griet De Cuypere, Robert Rubens et al., Voice problems in femaleto-male transsexuals, International Journal of Language and Communication Disorders 35 (2000), 3 , p. 427-442

Van Borsel/de Cuypere/Van den Berghe (2001): John van Borsel, Griet de Cuypere, Hilde van den Berghe, Physical appearance and voice in male-to-female transsexuals, Journal of Voice 15 (2001), 4, p. 570-575

Wendler et al. (1996): Jürgen Wendler, Wolfram Seidner, Gerhard Kittel et al., Lehrbuch der Phoniatrie und Pädaudiologie, Stuttgart 1996, S. 44-132 